\title{
Wide Area Ontology Integration Scheme for Reasoning Agents in Surveillance Networks
}

\author{
Soomi Yang ${ }^{*}$, Heejung Byun ${ }^{2}$ \\ ${ }^{1}$ Department of Information Engineering, the University of Suwon, Hwasung-si, Gyeonggido, Korea. \\ 2 Department of Information and Telecommunication Engineering, the University of Suwon, Hwasung-si, \\ Gyeonggi-do, Korea. \\ * Corresponding author. Tel: +82312298284; email: smyang@suwon.ac.kr \\ Manuscript submitted April 1, 2015; accepted December 20, 2015. \\ doi: $10.17706 /$ jcp.11.6.497-503
}

\begin{abstract}
In this paper an efficient and scalable surveillance network providing systematic collection and analysis of social security data based on distributed cooperative ontology framework is proposed. Upon ontology integration system, each reasoning agent can build and process ontology cooperatively. They share context ontology for cooperative combined inference. In the process of reasoning agents not only can get services from a region server but also can form and generate a P2P(peer-to-peer) overlay network to provide services to each other in wider bandwidth. Structured P2P overlay network forms useful substrate because it provides extendable, scalable, self-organizing and reliable framework. For efficient ontology integration the data weighting and similarity measure is incorporated and shows better performance.
\end{abstract}

Key words: Agent, context ontology, data weighting, peer-to-peer network surveillance networks.

\section{Introduction}

In wide area surveillance environment each surveillance device is equipped with a customized agent. Agents perform context reasoning to understand present circumstantial situation and derive related judgment utilizing ontologies. Context is any information that can be used to characterize the situation of environment entities. Ontologies are structured specifications of concepts, properties, and relationships that are required for understanding the context of an area. They contain customized knowledge for their specific environment of local area. For more easy and efficient cooperation between agents to achieve high level inference, a more efficient and scalable data management scheme is required. It should also utilize P2P computing and an adaptive cache scheme based on heterogeneous devices to support efficient use of network bandwidth.

To overcome the variety of surveillance devices which include cameras or sensors, agents for each device are built and interface each other through common interfaces. Each agent tries to be more highly context-aware through integration, analysis and inference of data for better decision. For superior inference, they need not only data of their own but also data of neighbor agents in near region. P2P computing utilizing common interface provides flexible, scalable and efficient ontology sharing method.

As a cooperative ontology integration scheme for heterogeneous devices and data network, caching and similarity measure techniques update and share data in efficient and cooperative way. Experiments and implementation are conducted to evaluate the effectiveness of suggested adaptive cache scheme.

The rest of the paper is organized as follows. In Section 2 cooperative ontology management framework 
for surveillance networks is suggested. Experiments and implementation are explained in Section 3. Section 4 reviews the related works on cooperative inference based on ontology and concludes this paper.

\section{Cooperative Ontology Management Framework}

\subsection{Collaboration Network between Agents}

Wide area surveillance networks construct a web of inference systems. Each surveillance data source server is equipped with an autonomous agent having local context ontology and inference engine. Ontologies model and annotate surveillance information and knowledge. They provide structured and manageable frameworks for modeling environmental knowledge and deriving context awareness which leads to proper response decision. Cooperative inference requires various domain knowledge necessary for advanced higher level inference including environmental information merging and alignment, event information association and matching, feature discovery and decision support based on information and data acquisition and integration. Data associations between data sources are required to cover larger areas to monitor multiple regions or locations. Our distributed cooperative inference framework manages ontology processing, ontology location and ontology connection on behalf of each agent.

Each data source agent communicates each other within access control permission. In the case of multi-agent systems, association of data across the data source servers is related to several levels of security requirements. Regulation of security levels is indispensable for efficiently controlled context awareness and decision making. And it provides security professionals can efficiently monitor and understand public security conditions with proper overhead.

The multimedia data and biometric feature information are distributed over the region servers and data source servers. The data source servers need to carry out the indexing and retrieval of the information distributed across the lowest level surveillance devices.

For each data request, data source server $n_{i}$ first tries to find the required data item from its local cache. If it encounters a local cache miss, it broadcasts a request message to its neighbor peers via P2P broadcast communication. The proposed ontology framework includes common API(Application Programming Interface)s for the operations. If a neighbor peer does not have the needed data item, the agent will send the request to the higher region server $m_{i}$ to obtain the needed data.

While peers exchange such information as the cache sizes of their neighbors and the transmission ranges, they can negotiate and come up with a cache strategy based on the messages exchanged. As a result, better usage of their limited cache space and higher system performance can be obtained

\subsection{Semantic Similarity Measure Incorporation}

The similarity measure is the semantic similarity between different ontologies of neighbor agents. For the better inference considering broader area ontologies of surroundings should be integrated. They should be merged and aligned according to the semantic similarity. Resulted smaller bounded ontologies also help efficient use of network bandwidth and enhance interoperability between agents.

There are several measures of semantic similarity [1]-[3]. They use the information content of the shared ancestors or descendants of the two terms. Similarity(concept1, concept2) is the set of common concepts shared by both concept 1 and concept 2 .

$$
\text { Similarity }\left(\text { Concept1, Concept2) }=\frac{\text { Metric * WeightedPathLength(CommonConcept(Concept1, Concept2) }}{\text { WeightedPathLength(Concept1) }+ \text { WeightedPathLength }(\text { Concept2) }}\right.
$$

As ontology allows multiple parents for each concept, two terms can share parents by multiple paths. 
Semantic distance is length of the paths. Furthermore weighted ontology refines boundary of resulting integrated ontology. Importance is assigned to different concepts and properties. Semantic distance values are analyzed to get semantic similarity measure. To validate the semantic similarity measures, the values are incorporated into the caching scheme.

To have fresh adapted information knowledge base, it should be merged, aligned, and integrated continuously. Adapted knowledge base helps inference intelligence and efficient network bandwidth use. Existing measurement algorithms for semantic similarity for use in inference are adopted including common ancestor based methods and common descendant based methods. These measurement algorithms are applied to the surveillance information content. We tested measures by analyzing semantic similarity. The more closely similar two sequences are, the more similar their ontological annotation is. As the bases for a search tool these measures are also used and operated over the ontological annotations.

To maintain the freshness and effectiveness of the data, data is cached adaptively and periodically. We measure the weight of cached data to describe its relative importance as compared to the other data as similar to [4]. The weight is computed as the product of the access frequency, size of the data, the data recency and the location distance in the number of hops, and it is customized proportionally to the similarity measure.

$$
\text { Weight }=\text { Freq }^{f} \text { DataSz }^{s} \text { Time }^{r} \text { Dist }^{d} \mu \text { SimilarityMeasure }^{m}
$$

The exponents are weighting factors. According to the weight computation, the content of cache is refreshed. As a result, the packet traffic for the cache management would be proportional to the probability of cache miss, latency, the probability of packet loss, the weight of data and the inverse of the similarity measure.

\section{Experiments and Implementation}

In a wide area cooperative surveillance environment, multimedia data and biometric feature information are produced continuously. Furthermore the multimedia data transmission consumes large network bandwidth. When the proposed adaptive caching technique is applied, experiments show better performance as shown in Fig. 1. E means the expected number of packets transmitted for normalized similarity measure $s$ and caching ratio $q$.

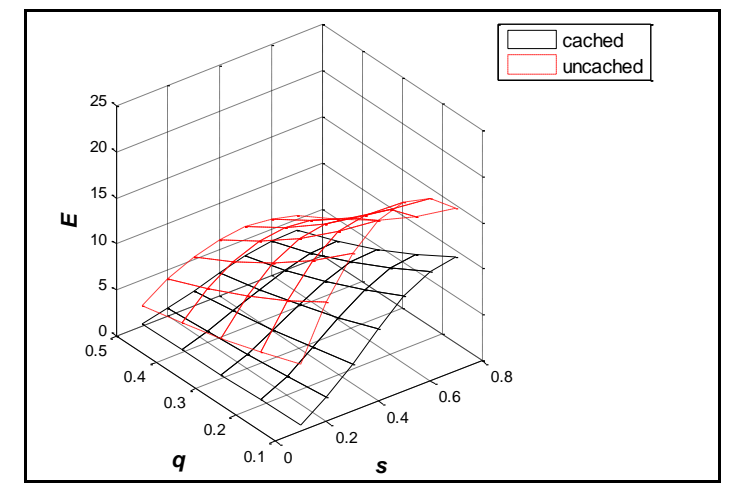

Fig. 1. Expected packet transmission comparison.

In the Fig. 1, the reduced number of packet transmission can be observed for the caching scheme proposed in Section 2. As the caching ratio $q$ increases, the number of packets transmitted is reduced. The data source servers might be grouped by region according to the administrative organization. The caching skewed by region could be incorporated through the variance in caching ratio. Although semantic similarity 
processing reduces the ontology size, semantically similar data incurs more data movement. These probabilities are reflected in Fig. 1.

Higher caching ratio $q$ reduces packet transmission $E$ as we expected. However increase in cache size of each agent caching provoke higher memory requirement in total system resulted in additional expenses. Proper cache size should be decided taking caching ratio, packet transmission and cost into consideration.

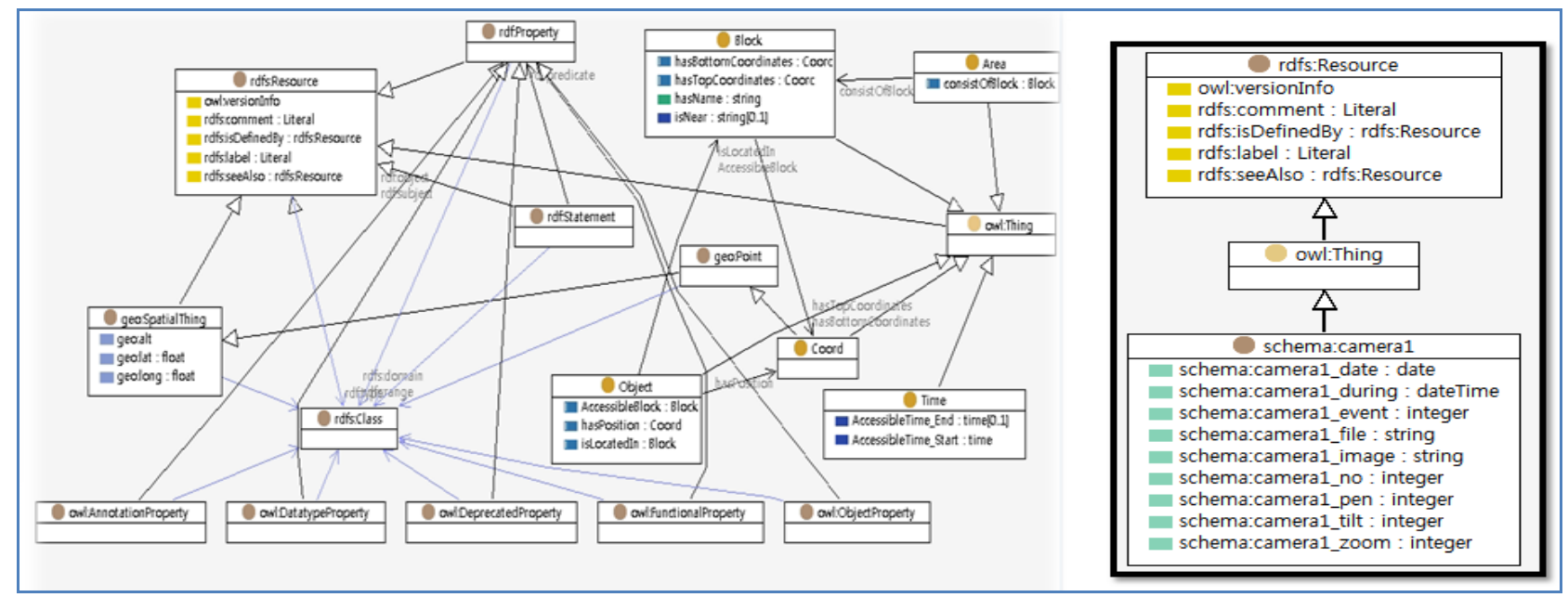

(a) Part of geography ontology

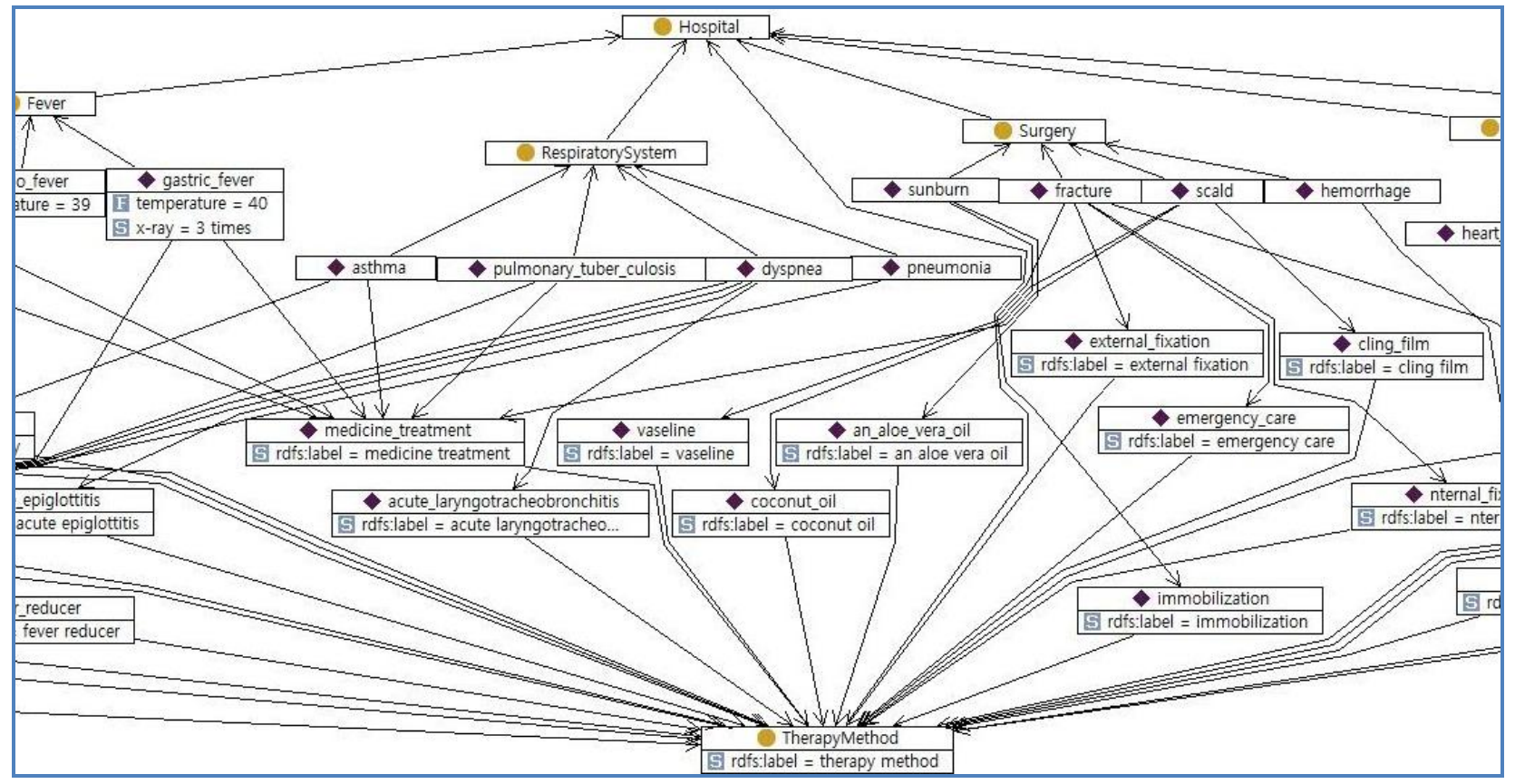

(b) Part of event ontology

Fig. 2. Part of ontology implemented.

To implement the proposed ontology integration scheme, context ontologies are built. Fig. 2 shows part of ontology implemented. For the integration of ontologies between data source servers especially for cameras, common interfaces according to the ONVIF standard [5] are implemented. Following ONVIF standard enhances interoperability between agents. In prototype system, identified events and feature data for context aware computing is structured for similarity measure into knowledge bases. To process ontology knowledge base the prototype system use SPARQL [6] with Jena [7] libraries.

Mobile user interface as well as web interface for administrators and users is developed. Sample mobile 
interface for iphone is shown in Fig. 3. (a) shows pop-up menu for each cameras on google map. (b) shows real-time streaming of camera. (c) shows cameras on the site scene. (d) shows push alarm for emergency. In addition it provides SNS(Social Network Service) liaison.

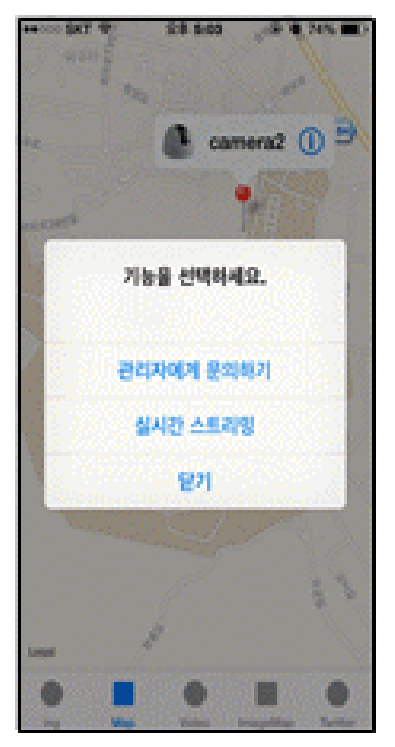

(a)

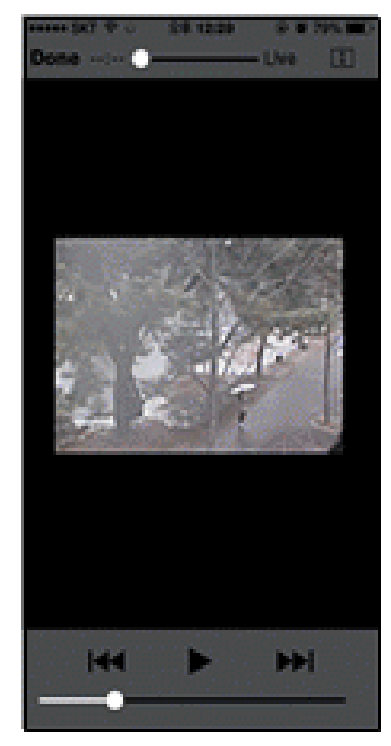

(b)

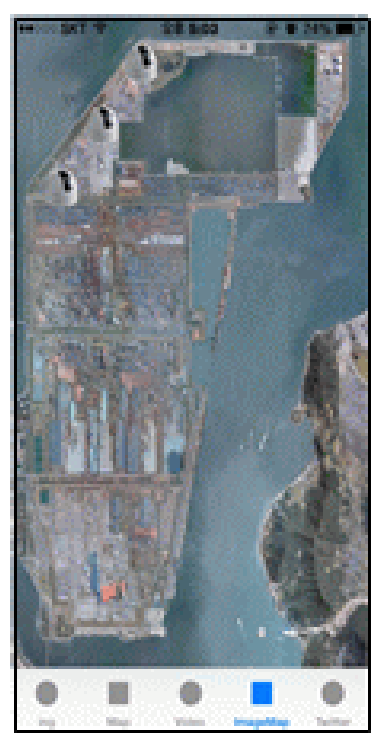

(c)

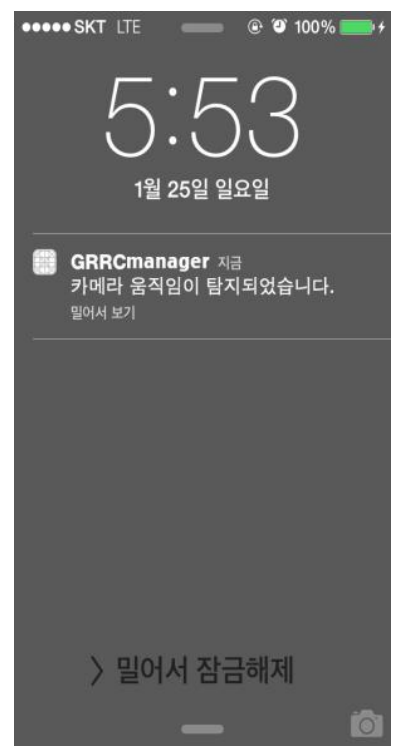

(d)

Fig. 3. Mobile interface for iphone.

For the secure data transmission and management encryption techniques are partially introduced. Security professionals can utilize it according to circumstances. Target implementation of designing and deploying a surveillance system will contain about 15 cities. Final product is to be included in the construction of the ubiquitous city.

\section{Related Works and Conclusion}

Several ontology reasoning systems have been developed for reasoning and querying the semantic web as seen in [1]-[3], [8]-[10]. They applied ontology to various environments. They tried to be context aware in several depth. However all of them do not provide common interfaces for communication for the integration or collaboration of dispersed ontology. Cooperative cache scheme for similar peers is described in [11]-[13], which combines the P2P communication technology with a conventional mobile system. However they do not apply their technique to the cooperative surveillance system. As the scope of surveillance system grows wider, it's network does not insist closed circuit anymore. It is based on Internet and required its own overlay network over it. Context inference including distributed multimedia data and feature data is widely used in surveillance including health surveillance as seen in [14]. Surveillance network for social security should also utilize it partly to care human disaster. Surveillance network equipped with reasoning agents having ontologies as proposed in this paper is required as a more secure and efficient practical model.

In this paper an efficient ontology management scheme for better context reasoning is proposed. Data acquisition servers communicate each other freely within access control permission. They perform intelligent cooperative inference based on integrated ontology knowledge base utilizing common APIs between them. It provides seamless real time tracing using various sensors, accurate location trace and technology fusion resulting in the value-added knowledge industry. Furthermore multiple data storage, query process, context knowledge base construction and knowledge processing for context-aware information system in efficient management scheme are provided. For the secure data transmission and 
management, encryption of data is applied partially. Data weighing and information similarity measure conformity to update and share data in a cooperative way enhance the data management performance. It reduces the packet transmission and helps the ontology integration efficiency.

\section{Acknowledgment}

This work was supported by the GRRC program of Gyeonggi province. [GRRCSUWON2014-B1, Center for U-city Security and Surveillance Technology].

\section{References}

[1] Ballatore, A., Bertolotto, M., \& Wilson, D. C. (2012). Geographic knowledge extraction and semantic similarity in open street map. Information Knowledge and Information Systems, 37(1), 61-81.

[2] Montserrat, B., David, S., \& Aida, V. (2011). An ontology-based measure to compute semantic similarity in biomedicine. Journal of Biomedical Informatics, 44(1), 118-125.

[3] Dheeraj, R., Erik, C., Daniel, O., \& Kenneth, K. (2013). A graph-based approach to commonsense concept extraction and semantic similarity detection. Proceedings of the 22nd International Conference on World Wide Web Companion (pp. 565-570).

[4] Paknikar, A., Kankanhalli, M., \& Ramakrishnan, K. (2000). A caching and streaming framework for multimedia. ACM Multimedia, 13-20.

[5] ONVIF (Open Network Video Interface Forum). (2014). Core Specification Version 2.4.2.

[6] W3C Recommendation. From http://www.w3.org/TR/rdf-sparql-query/

[7] Apache Jena. From http://jena.apache.org/

[8] Flahive, A., Rahayu, W., \& Taniar, D. (2005). A distributed ontology framework in the semantic grid environment. Proceedings of IEEE 19th International Conference on Advanced Information Networking and Applications (pp. 193-196).

[9] Pan, J. Z. (2007). A flexible ontology reasoning architecture for the semantic web. IEEE Transactions on Knowledge and Data Engineering, 19(2), 246-260.

[10] Anne, S., \& Heiner, S. (2008). Towards distributed ontology reasoning for the web. Proceedings of IEEE/WIC/ACM International Conference on Web Intelligence and Intelligent Agent Technology (pp. 536-539).

[11] Yin, L., \& Cao, G. (2006). Supporting cooperative caching in ad hoc networks. IEEE Transactions on Mobile Computing, 5(1), 77-89.

[12] Zheng, J., Su, J., \& Lu, X. (2005). A clustering based data replication algorithm in mobile ad hoc networks for improving data availability. Lecture Notes in Computer Science, 3358, 399-409.

[13] Chow, C., Leong, H., \& Chan, A. (2005). Distributed group-based cooperative caching in a mobile broadcast environment. ACM Proceedings of the 6th International Conference on Mobile Data Management (pp. 97-106).

[14] Shaban-Nejad, A., \& Haarslev, V. (2011). An enhanced graph-oriented approach for change management in distributed biomedical ontologies and linked data. Proceedings of the IEEE International Conference on Bioinformatics and Biomedicine Workshops (pp. 615-622).

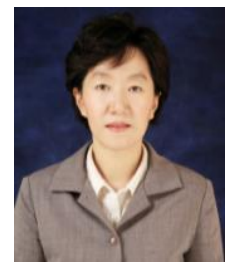

Soomi Yang received the B.S., M.S. and Ph.D. degrees in computer engineering from Seoul National University of Seoul, Korea, in 1985, 1987 and 1997 respectively. From 1988 to 2000, she was a researcher at Korea Telecom Research Center where she worked on telecommunication network, internet and information security. From 2000 to 2001, she was a visiting scholar at UCLA, USA. From 2002 to 2004, she was a faculty of the Suwon Science 
College. Since 2004, she has been on the Faculty of the University of Suwon, Korea, where she is a professor of computer sciences. Her research interests in information security include access control, network security, and secure system software.

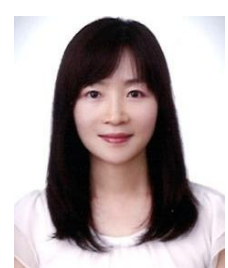

Heejung Byun received the B.S. degree from Soongsil University, Korea, in 1999, the M.S. degree from Korea Advanced Institute of Science and Technology (KAIST), Korea in 2001, and the Ph.D. degree from KAIST in 2005. She was a senior researcher in Samsung Electronics, Ltd. from 2007 to 2010. She is currently a professor with the Department of Information and Telecommunications Engineering, the University of Suwon, Korea. Her research interests include network protocol, network modeling, controller design, and performance analysis. 